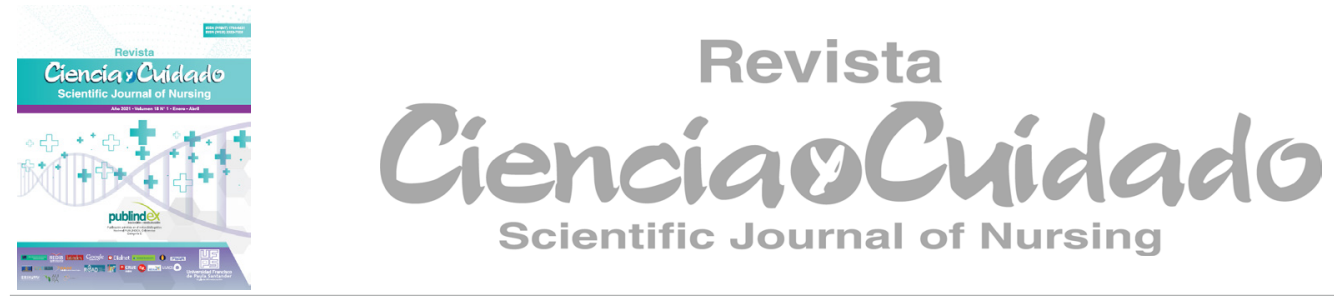

Articulo Original

\title{
Teoría de seres humanos unitarios en el cuidado de la administración de medicamentos
}

\author{
Unitary human beings theory in the care of the administration of medicines \\ Teoria do ser humano unitário no cuidado da administração de medicamento
}

\author{
Blanca Elpidia Tovar-Riveros ${ }^{1}$ \\ Omar Francisco Mena-Flórez ${ }^{2}$ \\ Johan Daniel Molano-Oviedo ${ }^{3}$
}

\section{Resumen}

La administración de medicamentos es uno de los pilares fundamentales en la gestión del cuidado de la enfermería, de ahí la importancia y responsabilidad de su manejo con personas que estén recibiendo fármacos especializados por su condición aguda o crítica. Objetivo: Construir un instrumento de valoración con los principios homeodinámicos y el concepto de pandimensionalidad de la teoría de seres humanos unitarios, para la administración de medicamentos especializados, como inotrópicos y vasopresores en servicios de UCI y urgencias. Materiales y métodos: Es una investigación cualitativa con enfoque descriptivo, para conocer la realidad del sujeto de cuidado desde la premisa sobre la relación de la administración de medicamentos y los principios de la teoría de Seres Humanos Unitarios. Resultados: Se construyó un instrumento de valoración desde la perspectiva de la teoría de Rogers. La misma permite al profesional de enfermería evaluar y generar propuestas de cuidado guiadas al equilibrio armónico de los campos de energía, según propone esta teórica en relación con la administración de medicamentos. También le facilita al estudiante el identificar las alteraciones de los principios de la teoría en relación a los efectos de los medicamentos. Conclusiones: El instrumento permite contemplar los principios homeodinámicos y la pandimensionalidad; además, facilita los procesos de cuidado en la administración de medicamentos, que en ocasiones por diferentes causas no se evalúa la respuesta del sujeto de cuidado ante un medicamento, retrasando su evolución ante la enfermedad.

Palabras clave: Enfermería de Cuidados Críticos, instrumento, valoración, procedimiento de tratamiento, descriptores de salud.
Autor de correspondencia*

${ }^{1 *}$ Enfermera. Especialista en Cuidado Crítico. Magister en Educación y Docencia Universitaria. Doctorante en Bioética. Docente Programa de Enfermería Fundación Universitaria del Área Andina. Email: btovar@ areandina.edu.co. Bogotá, Colombia. iD

${ }^{2}$ Estudiante Programa de Enfermería, Fundación Universitaria del Areandina. Email: omenaflorez@gmail.com. Bogotá, Colombia (iD)

${ }^{3}$ Estudiante Programa de Enfermería, Fundación Universitaria del Areandina. Email: danielmolano690@gmail.com. Bogotá, Colombia iD

Recibido: 23 abril 2020

Aprobado: 20 octubre 2020

Para citar este articulo:

Tovar-Riveros BE, Mena-Flores OF, Molano-Oviedo JD. Teoría de seres humanos unitarios en el cuidado de la administración de medicamentos. Rev. cienc. cuidad. 2021;18(1):30-41. https://doi. org/10.22463/17949831.2449

(C) Esta obra está bajo licencia internacional CC-BY-NC-ND (c) (1) $(9)$ 


\begin{abstract}
The administration of medications is one of the fundamental pillars in the management of nursing care, hence the importance and responsibility of the management of medications to people who are receiving specialized medicine for their acute or critical condition, that is why this study was proposed The objective: is to build an assessment instrument with the homeodynamic principles and the concept of pandimensionality of the theory of unitary human beings for the administration of specialized drugs such as inotropics and vasopressors in ICU and emergency health services. Materials and methods: qualitative with a descriptive approach, to know the reality of the care subject from the premise of the relationship of the administration of medicines and principles of the theory of Unitary Human Beings, Results: an assessment instrument was built from the perspective of Rogers' theory that allows the nursing professional to evaluate and generate proposals for care guided to the harmonic balance of the energy fields proposed by this theory in relation to the administration of medications, also, it allows the student to identify alterations in the principles of the theory in relation to the effects of medications Conclusions: the instrument allows to contemplate homeodynamic principles and the concept of pandimensionality thus facilitate the process of care for the administration of drugs that sometimes for different reasons does not evaluate the response of the care subject to a drug, delaying the evolution of the subject before the disease.
\end{abstract}

Keywords: Critical Care Nursing, Instrument, Valuation.

\title{
Resumo
}

A administração de medicamentos é um dos eixos fundamentais do cuidado de enfermagem, sendo importante a responsabilidade do seu manejo com pessoas que recebem fármacos especializados pela sua condição aguda ou crítica. Objetivo: Construir um instrumento de avaliação dos princípios homeodinâmicos e o conceito de pandimensionalidade da teoria do ser humanos unitários, para a administração de medicamentos especializados como inotrópicos e vasopressores nas UTI e Unidades de Emergência. Materiais e métodos: Pesquisa qualitativa com foco descritivo para conhecer a realidade do sujeito de cuidado sobre a relação da administração de medicamentos e os princípios da teoria do Ser Humano Unitário. Resultados: Construiu-se um instrumento de valoração desde a perspectiva de Rogers, permitindo ao profissional de enfermagem avaliar e gerar propostas de cuidado guiadas ao equilíbrio harmônico dos campos de energia, segundo a proposta da teoria em relação com a administração de medicamentos. Também lhe facilita ao estudante identificar as alterações dos princípios da teoria em relação com os efeitos dos medicamentos. Conclusões: O instrumento permite contemplar os princípios homeodinâmicos e pandimensionalidade; além disso, facilita os processos de cuidado na administração de medicamentos que ocasionalmente, por diferentes causas não se avalia a resposta do sujeito de cuidado ante um medicamento, retrasando a sua evolução perante a doença.

Palavras-chave: Enfermagem de Cuidados Críticos, teste, valoração, procedimento de tratamento, descritores em ciências da saúde.

\section{Introducción}

La enfermería como lo establece la Ley 266 de 1996 en el capítulo II "es una profesión liberal y una disciplina de carácter social, cuyos sujetos de atención son la persona, la familia y la comunidad, con sus caracte- rísticas socioculturales, sus necesidades y derechos, así como el ambiente físico y social que influye en la salud y en el bienestar (1)". En suma, es una ciencia de cuidado que desde sus inicios ha evolucionado y transformado a partir de su epistemología hasta las nuevas propuestas de cuidado para beneficiar e impactar en la recuperación de 
las personas, tal como lo muestra la historia, con la primera enfermera Florence Nightingale, quien transformó la enfermería y la hizo visible ante el mundo. Durante años estas enfermeras han sido símbolos importantes para dicha innovación por su contribución con teorías que esbozan un ejercicio práctico del cuidado hacia la persona en todas sus dimensiones, desde sus formas de pensar hasta en los entornos que la rodean. Martha E. Rogers, autora que incursionó con su Teoría de Seres Humanos Unitarios en el año 1970 explicó cómo el ser humano está relacionado consigo mismo, los demás y el entorno. Esta autora analizó el ser humano a partir de los campos de energía de sistemas abiertos, basado en tres principios homeodinámicos y en el concepto de pandimensionalidad que permiten evaluar el cambio del estado de la persona tanto físico, como mental y social. El primero que corresponde a la resonancia se refiere a la capacidad de respuesta de la persona ante un estímulo, que para esta investigación es la administración de medicamentos. El segundo, que es la integralidad valora la totalidad de todas las dimensiones del individuo. El tercero, la helicidad evalúa la evolución del estado de la persona. Por otra parte el concepto de pandimensionalidad relaciona la individualidad de cada persona con sus diferentes dimensiones: emocional, física y espiritual entre otras (2).

Rogers definió a la persona como un sistema abierto, un "campo de energía irreductible, indivisible y pandimensional" (3). Por lo tanto, el cuidado para la persona es único e individual, pues cada ser humano es diferente y sus patrones o potenciales de salud son irrepetibles. En concordancia con lo planteado, los profesionales de enfermería deben generar planes de cuidado acordes a la respuesta humana, por tanto no pueden ser generalizados ni iguales para todos (3).

Cada uno de los principios homeodinámicos de la teoría son considerados como procesos vitales que se dividen en dominios y patrones que pueden ser evaluados mediante el proceso "causa y efecto", que manifiesta el individuo a través de respuestas en sus signos vitales, cambios en aspectos físicos, emocionales, mentales y sociales. Con respecto a lo planteado anteriormente, es que surge este instrumento para permitir que los estudiantes de enfermería generen planes de cuidado en la administración de medicamentos, teniendo en cuenta los principios homeodinámicos y la pandimensionalidad expuestos en la teoría de seres humanos unitarios de Martha E. Rogers, a personas que por su estado crítico o agudo estuviesen recibiendo medicamentos espe- cializados.

Por ello, es importante describir los parámetros del instrumento y la relación con estos medicamentos (vasopresores e inotrópicos). Un instrumento de valoración es una herramienta que permite conocer la percepción de bienestar de las personas ante una situación específica (4).

Dicho lo anterior, es necesario preguntarse ¿Es posible aplicar los principios homeodinámicos y la pandimensionalidad de la teoría de seres humanos unitarios en la administración de medicamentos vasopresores e inotrópicos, como herramienta de valoración de enfermería? Interrogante que se responde teniendo en cuenta que es una tarea propia de la enfermería y que cada persona es particular en todos sus entornos; por tanto hay que vigilar la administración de medicamentos y todos sus cuidados. La investigación siguió los parámetros éticos según la Resolución 8430 de 1993 (5) y como hace parte de un proyecto institucional cuenta con la aprobación del comité de ética del hospital donde se aplicará para verificar su confiabilidad.

\section{Objetivo}

Construir un instrumento de valoración con los principios homeodinámicos y el concepto de la pandimensionalidad de la teoría de seres humanos unitarios, para la administración de medicamentos especializados como inotrópicos y vasopresores en servicios de salud de UCI y urgencias

\section{Materiales y Métodos}

Esta investigación se realizó con un enfoque cualitativo-descriptivo, dado que permite al investigador resolver hipótesis que permitan el desarrollo de una problemática. Se seguirán las siguientes fases que permiten: el desarrollo de la idea, planteamiento del problema, revisión bibliográfica, desarrollo del diseño de investigación y elaboración del reporte de resultados del fenómeno que se desea investigar (6).

\section{Esta investigación se desarrolló en fases:}

Fase 1: se realizó una revisión de literatura para el análisis de la información sobre medicamentos y la teoría de Rogers. El análisis se llevó a cabo mediante una matriz en Excel. Se tuvieron en cuenta los siguientes criterios de inclusión: artículos académicos y científicos, 
documentos, escritos y trabajos de grado en idiomas español, inglés y portugués; se determinó que el 20\% de las fuentes teóricas sean mayores a los 5 años y el $80 \%$ restantes sean menores a los 5 años. También, se indagó en otros idiomas, artículos académicos y científicos, documentos, escritos y trabajos de grado que contengan información clave con relación a la teoría de Martha E. Rogers y la administración de medicamentos especializados. Como criterios de exclusión: documentos que contengan referencias de otras teóricas en enfermería, medicamentos que no sean especializados.

Fase 2: Se construyó un instrumento a partir de los principios homeodinámicos y el concepto de la pansimensionalidad que expone la teoría, para categorizar las respuestas humanas, asignando un valor numérico que indica el estado armónico de los campos energéticos del individuo relacionados con la administración de los medicamentos. El instrumento se estructuró a partir de los tres principios y el concepto de pandimensionalidad de la teoría; además, se asignó un valor numérico de 0,1 y 2 dependiendo de la respuesta humana a la variable valorada. Se evalúa el $0=a$ nada, $1=$ moderada $y$ $2=\mathrm{a}$ alta, para un total de los 4 principios de 60 puntos.

Fase 3: se realizó la revisión del contenido del instrumento para contrastarlo; posteriormente se realizaron ajustes, mediante una lista de chequeo valorada por expertos en la temática. Finalmente se efectuaron los ajustes en cuanto a redacción, conceptos, categorización, patrones y dominios a valorar.

\section{Resultados}

Estos se presentan en tres etapas: la primera corresponde a la fase 1, que evidenció la relación de la teoría de Rogers o Seres Humanos Unitarios con la administración de medicamentos especializados; la fase 2, permitió la construcción del instrumento con base en los principios homeodinámicos y el concepto de la pandimensionalidad de la teoría; y, en la fase 3, el instrumento fue evaluado por docentes expertas en la temática y la teoría a partir de lista de chequeo.

Fase 1: Relación vasopresores e inotrópicos con los principios homeodinámicos de la teoría

\section{vasopresores}

Se componen principalmente de catecolaminas naturales o también conocidas como fármacos simpaticomi- méticos, pues imitan la función del sistema nervioso simpático a través del reemplazo de estas sustancias que son segregadas naturalmente en la médula adrenal y que actúan en los diferentes receptores adrenérgicos (A1, A2, B1, B2) y dopaminérgicos; además, son receptores de vasopresina, que es un vasopresor. Todos causan principalmente efectos excitatorios (vasoconstricción) (7-8). Dentro de este grupo se encuentran:

La Noradrenalina que estimula a receptores Alfa 1 y hace vasoconstricción, aumentando la tensión arterial, mejora el gasto cardiaco (7-9); con relación al principio hemodinámico Helicidad este puede aumentar la frecuencia cardiaca y mejorar las cifras tensionales del paciente; como efecto inverso puede ocasionar hipotensión, isquemia de órgano y cambios hemodinámicos negativos.

La Adrenalina tiene su acción en los receptores Alfa y Beta ocasionando mejoría de gasto cardíaco y ventilación Evoluciona corazón, bronquios y vasos sanguíneos (7-9). Con relación al principio hemodinámico de la Helicidad este produce elevación de presión arterial, frecuencia cardiaca y mejoría de patrón ventilatorio; como consecuencia adversa puede provocar Hipotensión, arritmias y signos de shock (palidez, frialdad).

La Dopamina cumple su función como precursora de la noradrenalina y es estimuladora de los receptores alfa beta adrenérgicos y dopaminérgicos, dependiendo de su dosis de infusión varía el efecto terapéutico (9-11). Con relación al principio hemodinámico Helicidad este produce elevación de presión arterial, frecuencia cardiaca y mejoría de patrón ventilatorio; también puede producir hipotensión y arritmias cardiacas.

La Vasopresina es una hormona secretada por la hipófisis y mediada por los barorreceptores. Actúa en receptores propios: V1 vascular, donde causa su principal efecto vasoconstrictor, aumentando la concentración del calcio intracelular y V2 renal que genera retención de sodio y agua (7-9). Con relación al principio hemodinámico Helicidad este sube la presión arterial y mejora el gasto cardiaco en dosis máxima, sin objetivo terapéutico puede ocasionar efectos adversos como la hipotensión.

\section{Inotrópicos}

Aumentan la contractilidad cardiaca para mejorar el gasto cardíaco a través de diferentes mecanismos de acción, pero principalmente interviniendo en el ion calcio y en el potencial de acción de la célula miocárdica, con- 
tribuyendo así a la estabilidad hemodinámica (7-11). Dentro de este grupo se encuentran:

La Milrinona Amrinona son inhibidores de la fosfodiesterasa 3 (FD 3), que impiden específicamente la acción de esta enzima FD3, manteniendo así la concentración de calcio intracelular y generando vasodilatación pulmonar y periférica $(8,10,11)$. Con relación al principio hemodinámico Helicidad aumenta la frecuencia cardíaca y mejora el funcionamiento de órganos; como efecto adverso puede causar signos de shock y falla cardíaca.

El Levosimendan es considerado un sensibilizador de los canales del calcio y tiene dos funciones: sensibilizar el miocito al calcio a través de su unión a la troponina $\mathrm{C}$ y hacer apertura de los canales de potasio $(8,10,11)$. Con relación al principio hemodinámico Helicidad aumenta la frecuencia cardíaca y mejora el funcionamiento de órganos; como efecto adverso es posible una falla cardiaca.

La Dobutamina es una catecolamina sintética, actúa principalmente en receptores beta adrenérgicos $\mathrm{B} 1$ y B2: B1 aumentando la contracción cardiaca y en los receptores $\mathrm{B} 2$ del músculo liso generando vasodilatación $(8,10,11)$. Con relación al principio hemodinámico Helicidad aumenta la frecuencia cardíaca y mejora la función de órganos; como efecto adverso por dosis inadecuadas produce taquiarritmias e hipotensión.

Tanto inotrópicos como vasopresores intervienen en el estado hemodinámico del paciente; unos intervienen sobre las propiedades cardiacas y otros a nivel periférico. Por tanto, se espera que evolucione el corazón como bomba, aumentado su contracción; los vasos sanguíneos a través del cambio de su tono; y, por último, la funcionalidad de los órganos al obtener mejor perfusión por medio de la volemia sanguínea expulsada por el corazón y transportadora de oxígeno a través de dicho sistema vascular (12-13).

Sistemas relacionados en la administración de medicamentos

\section{Piel}

Con relación al principio homeodinámico, su Integralidad valora el impacto (osmolaridad) y la técnica (catéter), así como el estado óptimo del catéter y la vía de acceso vascular de administración y dosis.

\section{Estado Metabólico}

Con relación al principio hemodinámico Integralidad se valora la glucosa con respecto al aumento en valores $\mathrm{mg} / \mathrm{dl}$ de glucosa en sangre durante la terapia con inotrópicos y el peso en caso de que haya pérdida de peso por aumento del metabolismo. La administración de inotrópicos y vasopresores debe ser por vía central, debido a la alta viscosidad de estos fármacos y por el mismo mecanismo de acción sobre los vasos sanguíneos. Si se realiza una infusión directa por vía periférica aumenta el riesgo de necrosis de la extremidad, aunque en situaciones de urgencia es posible administrar por esta vía periférica mientras se consigue una central (14).

No obstante, unos de los efectos adversos, especialmente de las catecolaminas son los aumentos del consumo de oxígeno y cambios en el metabolismo por glucólisis, glucogenólisis, lipolisis, neoglucogénesis, cetogénesis, la resistencia periférica a la insulina y la liberación de lactato (7).

\section{Respuestas a la administración de medicamentos con relación al principio hemodinámico Resonancia}

Dentro de este principio se valora el tono muscular, los efectos adversos causados por el medicamento y el estado mental del paciente, evidenciados en los movimientos corporales, los cambios hemodinámicos y la escala de glasgow. Se considera que la mayoría de los pacientes con soporte vasopresor y en unidades de UCI y urgencias, tienen comprometido su estado de conciencia (13); por tanto, no pueden realizar actividades físicas de la vida diaria, involucrando su sistema osteomuscular.

\section{Dimensiones de la persona (pandimensionalidad)}

Se valora la relación que tenga el paciente y su familia, el cuidador (enfermera/o) y su entorno conformado por redes de apoyo familiares que contribuyan a la recuperación de la enfermedad; igualmente, se debe tener en cuenta el cuidado generado por la/el enfermero/a si es íntegro y humanizado; además, hay que observar el estado de acoplo que tenga el paciente respecto al ambiente hospitalario. Conviene subrayar que el ser humano es como un prisma visto desde diferentes aristas, siendo su familia un entorno fundamental para conseguir el equilibrio, con la cual intercambia energía en su diario vivir; además, está el personal de enfermería que actúa como primera línea de contacto y con el que interactúa durante su estancia hospitalaria y en su tratamiento, así como su entorno (habitación y/o cubículo) que se vuelve su hogar mientras logra recuperar el potencial de su salud

Fase 2. Construcción del instrumento a partir de los principios homeodinámicos de la teoría de Seres Hu- 


\section{manos Unitarios}

Un instrumento de medición es aquel que posibilita el registro de datos que representan variables para definir un plan de cuidados (4). Esta herramienta consta de cuatro categorías que hacen referencia a los principios y el concepto de la pandimensionalidad. Cada categoría se compone de subcategorías a las que se les asignó tres puntuaciones de 0,1 y 2 : baja que corresponde a un puntaje de 0 , moderada que da un puntaje de 1 y una puntuación alta de 2 para un total de 60 puntos. Se evalúan como sigue: de 41 a 60 puntos, significa que hay energía, equilibrio e interacción alta entre los campos; de 21 a 40 puntos, es equilibrio, interacción y energía moderada entre los Campos de energía; menor de 20 puntos, corresponde a baja energía, interacción y equilibrio entre los campos de energía. a) Relación del primer principio homeodinámico (helicidad) desde la perspectiva de los sistemas

En la tabla $\mathrm{N}^{0} 1$ se muestran los sistemas en los que actúa este tipo de medicamentos para determinar su influencia sobre: tensión arterial, tensión arterial media, frecuencia cardiaca, frecuencia respiratoria, el aporte de oxígeno y el estado renal del sujeto de cuidado. Es decir, que se quiere evaluar si la persona con estos medicamentos mejora, y así, establecer la evolución que hace referencia a la helícidad del campo de energía de la persona. Este instrumento permite observar la relación del primer principio homeodinámico de la teoría con los sistemas que podrían verse más beneficiados de este tipo de medicamentos. Esta primera evaluación corresponde a un puntaje parcial de 28 puntos que refleja un estado armónico del sujeto.

Tabla 1. Helícidad y sistemas relacionados con medicamentos especializados

\begin{tabular}{|c|c|c|c|c|c|c|c|}
\hline \multirow{2}{*}{\multicolumn{4}{|c|}{$\begin{array}{l}\text { Nombre del paciente: } \\
\text { Medicamentos administrados o en infusión: }\end{array}$}} & \multicolumn{4}{|c|}{ Patología actual del paciente: } \\
\hline & & & & & & & \\
\hline \multicolumn{4}{|c|}{ Fecha de valoración: } & \multicolumn{4}{|l|}{ Diagnóstico de enfermería: } \\
\hline $\begin{array}{l}\text { Ca mp o s } \\
\text { gener- }\end{array}$ & $\begin{array}{l}\text { Descrip- } \\
\text { ción del } \\
\text { campo }\end{array}$ & & & \multicolumn{3}{|c|}{ Puntuación del patrón } & \multirow{4}{*}{$\begin{array}{l}\text { Punta- } \\
\text { je por } \\
\text { patron }\end{array}$} \\
\hline \multirow[t]{18}{*}{ Helicidad } & \multirow{18}{*}{$\begin{array}{l}\text { Evolución } \\
\text { o cambios } \\
\text { continuoos } \\
\text { o perma- } \\
\text { nentes en } \\
\text { los proce- } \\
\text { sos vita- } \\
\text { les. }\end{array}$} & \multirow{3}{*}{$\underset{\text { Valorar }}{\text { Dominio }}$} & \multirow[t]{3}{*}{ Energía a Valorar } & \multicolumn{4}{|c|}{ Puntuación } \\
\hline & & & & Baja & Moderada & Alta & \\
\hline & & & & $\mathbf{0}$ & 1 & 2 & \\
\hline & & \multirow{4}{*}{$\begin{array}{l}\text { S i s t e m a } \\
\text { Cardiovas- } \\
\text { cular }\end{array}$} & Presión Arterial & $<=80 / 60 \mathrm{mmHg}$ & $<=100 / 80 \mathrm{mmHg}$ & $<=130 / 90 \mathrm{mmHg}$ & \\
\hline & & & Presión Arterial Me- & $<=40 \mathrm{mmHg}$ & $<=70 \mathrm{mmHg}$ & $<=110 \mathrm{mmHg}$ & \\
\hline & & & F.C & $<=40$ Latidos por minuto & $<=60 \underset{\text { nuto }}{\text { Latidos por mi- }}$ & $<=100 \begin{array}{c}\text { Latidos por mi- } \\
\text { nuto }\end{array}$ & \\
\hline & & & $\begin{array}{c}\text { Caracteristicas del } \\
\text { pulo }\end{array}$ & Filiforme & Irregular & Rítmico & \\
\hline & & \multirow{2}{*}{$\begin{array}{l}\text { S is te m a } \\
\text { Respirato- } \\
\text { rio }\end{array}$} & F.R. & $<=15 \begin{array}{c}\text { Respiraciones por } \\
\text { minuto }\end{array}$ & $<=18 \underset{\text { Respiraciones por }}{\text { minuto }}$ & $<=22 \begin{array}{c}\text { Respiraciones por } \\
\text { minuto }\end{array}$ & \\
\hline & & & Soporte de $\mathrm{O} 2$ & Sistema de alto flujo & Sistema de bajo flujo & Medio ambiente & \\
\hline & & \multirow{4}{*}{$\begin{array}{l}\text { Estado Hé- } \\
\text { modinámi- } \\
\text { co }\end{array}$} & $\mathrm{SaO} 2$ & $<70 \%$ & $<85 \%$ & $85-90 \%$ & \\
\hline & & & $\mathrm{T}^{\circ}$ & $<=33^{\circ}$ & $<=35^{\circ}$ & $<=37^{\circ}$ & \\
\hline & & & Coloración de la piel & Cianótica & Pálida & Normal & \\
\hline & & & Llenado Capilar & $<3$ segundos & 2-3 segundos & $<2$ segundos & \\
\hline & & \multirow[t]{5}{*}{$\begin{array}{l}\text { Sistema Re- } \\
\text { nal }\end{array}$} & Eliminación & Terapias $\underset{\text { renal }}{\text { deemplazo }}$ & $\begin{array}{c}\text { Sonda Vesical o Cistos- } \\
\text { tomía }\end{array}$ & Espontánea & \\
\hline & & & Gasto Urinario & $<0.5 \mathrm{ml} / \mathrm{kg} / \mathrm{h}$ (Anuria) & $0.6-0.9 \mathrm{ml} / \mathrm{kg} / \mathrm{h}$ (Oligu- & $1 \mathrm{ml} / \mathrm{kg} / \mathrm{h}$ (Normal) & \\
\hline & & & Azoados & Alterados sin Corrección & Alterados en Corrección & Rangos Normales & \\
\hline & & & Edema & Grado III y IV & Grado I y II & Sin edema & \\
\hline & & & & & Puntaje total por campo e & nergético sobre 28 puntos: & \\
\hline
\end{tabular}

Fuente: Elaboración propia 
b) Relación del segundo principio homeodinámico (integralidad) desde la perspectiva de la dimensión física.

En la tabla $\mathrm{N}^{\circ} 2$ se muestra cómo una persona o sujeto en cuidado crítico o estado agudo necesita una serie de aditamentos para armonizar su equilibrio hemodiná- mico; es así que para administrar los medicamentos se requiere de la valoración de la dimensión física como: el manejo de accesos, ingreso de líquidos y la función metabólica en relación al reposo para conservar la integridad de la piel. Esta segunda evaluación parcial corresponde a un puntaje de 14 puntos

Tabla 2. Integralidad y dimensión física

\begin{tabular}{|c|c|c|c|c|c|c|c|}
\hline & \multirow{9}{*}{$\begin{array}{l}\text { Visión unitaria } \\
\text { del sujeto de } \\
\text { cuidado desde } \\
\text { sus dimensiones } \\
\text { física. }\end{array}$} & \multirow[t]{2}{*}{$\begin{array}{c}\text { Dominio a va- } \\
\text { lorar }\end{array}$} & \multirow[t]{2}{*}{ Patrón a valorar } & \multicolumn{2}{|r|}{ Moderada } & Alta & \multirow[t]{2}{*}{$\begin{array}{l}\text { Puntaje por pa- } \\
\text { tron }\end{array}$} \\
\hline & & & & 0 & 1 & 2 & \\
\hline & & Acceso Venoso & Tipo de catéter & $\begin{array}{l}\text { Catéter Venoso } \\
\text { periférico }\end{array}$ & $\begin{array}{lr}\text { Catéter } & \text { Venoso } \\
\text { Central } & \text { Peri- } \\
\text { férico } & \text { (PICC, } \\
\text { DRUM, KAVA- } \\
\text { FIX) }\end{array}$ & $\begin{array}{l}\text { Catéter Venoso } \\
\text { Central. }\end{array}$ & \\
\hline & & Infusión de mez- & Flebitis & Bacteriana & Química o Física & No hay flebitis & \\
\hline & & & $\begin{array}{l}\text { Número de infu- } \\
\text { siones }\end{array}$ & $>=3$ Infusiones & 2 infusiones & 1 infusión & \\
\hline & & & $\begin{array}{l}\text { Dosis de infu- } \\
\text { siones }\end{array}$ & Máxima & Media & Mínima & \\
\hline & & & Glucometría & $\begin{array}{l}<50 \quad \mathrm{mg} / \mathrm{dl} \quad \mathrm{a} \\
>140 \mathrm{mg} / \mathrm{dl}\end{array}$ & $\begin{array}{l}<70 \text { a } 50 \mathrm{mg} / \mathrm{dl} \\
\text { o }>110 \mathrm{mg} / \mathrm{dl} \mathrm{a} \\
140 \mathrm{mg} / \mathrm{dl}\end{array}$ & $70-110 \mathrm{mg} / \mathrm{dl}$ & \\
\hline & & $\begin{array}{l}\text { Estado Metabó- } \\
\text { lico }\end{array}$ & Estado de la piel & $\begin{array}{l}\text { Presencia de úl- } \\
\text { ceras }\end{array}$ & $\begin{array}{l}\text { Perdida de tur- } \\
\text { gencia }\end{array}$ & Hidrata e integra & \\
\hline & & & Peso & $\begin{array}{l}\text { Perdió más de } \\
5 \mathrm{Kg}\end{array}$ & Perdió $5 \mathrm{Kg}$ & No perdió peso & \\
\hline Integralidad & & & & Puntaje total $\mathrm{p}$ & or campo energétic & o sobre 14 puntos: & \\
\hline
\end{tabular}

Fuente: Elaboración propia

c) Relación del tercer principio homeodinámico (resonancia o respuesta) desde la perspectiva física y mental

En la tabla $\mathrm{N}^{\circ} 3$ se presentan el sistema osteomuscular y el sistema nervioso (estado mental, movimientos), para comprender la respuesta del sujeto de cuidado al medicamento, además de un posible efecto adverso a la sustancia medicamentosa. Esta tercera evaluación parcial corresponde a un puntaje de 10 puntos.

Tabla 3. Resonancia y efectos secundarios

\begin{tabular}{|c|c|c|c|c|c|c|c|}
\hline \multirow{7}{*}{ Resonancia } & \multirow{7}{*}{$\begin{array}{l}\text { Efectos fisioló- } \\
\text { gicos secunda- } \\
\text { rios a los cam- } \\
\text { bios continuos } \\
\text { o permanentes }\end{array}$} & \multirow{2}{*}{$\begin{array}{c}\text { Dominio a va- } \\
\text { lorar }\end{array}$} & \multirow[t]{2}{*}{ Patrón a Valorar } & Baja & Media & Alta & \multirow[t]{4}{*}{ Puntaje por patron } \\
\hline & & & & 0 & 1 & 2 & \\
\hline & & \multirow[t]{2}{*}{$\begin{array}{l}\text { Estado osteo- } \\
\text { muscular }\end{array}$} & $\begin{array}{r}\text { Movimientos corpo- } \\
\text { rales }\end{array}$ & & & & \\
\hline & & & Tono Muscular & & & & \\
\hline & & Medicamentos & Efectos adversos & & & & \\
\hline & & \multirow[t]{3}{*}{ Estado Mental } & Glasgow & & & & \\
\hline & & & Nivel de conciencia & & & & \\
\hline & & & Pur & otal po & energétic & $10 \mathrm{pur}$ & \\
\hline
\end{tabular}

Fuente: Elaboración propia

d) Relación del concepto (pandimensionalidad) desde la perspectiva del entorno

En la tabla 4 se observa la adaptación del concepto de Rogers, tomándose en cuenta categorías como: la fa- milia, el rol del profesional de enfermería y el entorno donde se encuentra la persona o sujeto de cuidado, dado que esta dimensión influye en su recuperación. Esta cuarta evaluación parcial corresponde a un puntaje de 8 puntos. 
Tabla 4. La pandimensionalidad desde la perspectiva del entorno

\begin{tabular}{|c|c|c|c|c|c|c|c|}
\hline \multirow[t]{5}{*}{$\begin{array}{c}\text { Pan dimensiona- } \\
\text { lidad }\end{array}$} & \multirow{5}{*}{$\begin{array}{l}\text { Visión del sujeto } \\
\text { de cuidado des- } \\
\text { de la perspectiva } \\
\text { social, familiar, } \\
\text { emocional, poli- } \\
\text { tica y cultural. }\end{array}$} & \multirow{3}{*}{$\begin{array}{c}\text { Dominio a eva- } \\
\text { luar } \\
\text { Familia }\end{array}$} & Patrón a evaluar & \multirow{2}{*}{$\begin{array}{c}\text { Baja } \\
0\end{array}$} & \multirow{2}{*}{$\begin{array}{c}\text { Media } \\
1\end{array}$} & \multirow{2}{*}{$\begin{array}{l}\text { Alta } \\
2\end{array}$} & \multirow[t]{4}{*}{$\begin{array}{l}\text { Puntaje por pa- } \\
\text { trón }\end{array}$} \\
\hline & & & $\begin{array}{l}\text { Recibe visitas } \\
\text { Familiares }\end{array}$ & & & & \\
\hline & & & $\begin{array}{l}\text { Estado emocio- } \\
\text { nal de la familia }\end{array}$ & $\begin{array}{l}\text { Fases de depre- } \\
\text { sión y llanto, } \\
\text { refiere sentirse } \\
\quad \text { estresada }\end{array}$ & $\begin{array}{l}\text { Refiere preocu- } \\
\text { pación, no se } \\
\text { observa mejoría } \\
\text { del paciente }\end{array}$ & $\begin{array}{l}\text { Refiere sentir- } \\
\text { se tranquila por } \\
\text { evolución del } \\
\text { paciente }\end{array}$ & \\
\hline & & $\begin{array}{l}\text { Personal de en- } \\
\text { fermería }\end{array}$ & $\begin{array}{l}\text { Concepto de la } \\
\text { enfermera según } \\
\text { su valoración }\end{array}$ & $\begin{array}{l}\text { Refiere que no } \\
\text { hay evolución } \\
\text { ni respuesta al } \\
\text { tratamiento por } \\
\text { parte del pacien- } \\
\text { te }\end{array}$ & $\begin{array}{l}\text { Refiere mínima } \\
\text { evolución y res- } \\
\text { puesta al trata- } \\
\text { miento }\end{array}$ & $\begin{array}{l}\text { Refiere óptima } \\
\text { evolución con el } \\
\text { tratamiento }\end{array}$ & \\
\hline & & $\begin{array}{l}\text { Unidad, habita- } \\
\text { ción y/o cubí- } \\
\text { culo }\end{array}$ & Aislamiento & $\begin{array}{l}\text { Aislamiento Es- } \\
\text { tricto (Aerosol, } \\
\text { gotas, contacto) }\end{array}$ & $\begin{array}{l}\text { Aislamiento pro- } \\
\text { tector }\end{array}$ & $\begin{array}{c}\text { No se encuentra } \\
\text { aislado }\end{array}$ & \\
\hline & & & \multicolumn{5}{|c|}{ Puntaje total por campo energético sobre 8 puntos: } \\
\hline & & & & & Puntaje tota & 1 sobre 60 puntos: & \\
\hline
\end{tabular}

Fuente: Elaboración propia.

\section{Fase 3: Revisión para ajustes del instrumento}

Esta evaluación se hizo por parte de tres docentes que han trabajado sobre la teoría de Seres Humanos Unitarios. Se les envió el instrumento para que lo evaluaran en función de: objetivo, instructivo de diligenciamiento e interpretación del mismo. Se construyó con los cuatro principios de la teoría como se describió anteriormente. En la tabla $\mathrm{N}^{\mathrm{0}} 5$ se explican las sugerencias y cambios en aspectos a mejorar como: gramática, forma de enunciar el verbo, espacio donde se escribe el diagnóstico de enfermería y la motivación para la utilización en la valoración.

Tabla 5. Evaluación del instrumento por expertas en la teoría

\begin{tabular}{ll}
\hline \multicolumn{1}{c}{ Aspectos a evaluar } & \multicolumn{1}{c}{ Observaciones } \\
Gramática & Cambio de palabras, enunciación de verbos y terminología \\
Principios homeodinámicos y el concepto de & Los ven presentes, sugirieron ampliar la explicación del concepto y colocar \\
pandimensionalidad de la teoría & el diagnóstico de enfermería para su fácil relación e interpretación \\
Variables del instrumento & Pertinentes, se ajustaron unas sugerencias \\
Instructivo para diligenciamiento & Debe ser más claro para el fácil desarrollo \\
Incentivo para el uso del instrumento & Sugieren explicar en el instructivo cual sería la motivación para usarlo en la \\
& valoración de sujetos de cuidado. \\
Pertinencia & Se necesita este tipo de instrumento para evidenciar la relación de la teoría \\
& con la práctica \\
\hline
\end{tabular}

Fuente: autores

\section{Discusión}

La enfermería moderna se ha centrado en el cuidado íntegro y de calidad del paciente que garantice procesos seguros. Dentro de la normatividad que reglamenta la profesión de enfermería, está la ley 266 de 1996, en la que se expresa la individualidad que asegura un cuidado de enfermería que tiene en cuenta característi- cas sociales, comunitarias, familiares, físicas, mentales y emocionales de la persona. Igualmente, ayuda al enfermero a comprender el entorno que rodea al individuo; por tanto, le permite generar procesos de cuidado individuales humanizados que promuevan la salud del paciente (1). Desde la perspectiva Rogeriana la persona como ser único debe ser vista de una manera distinta a los demás. Según Rogers todas las personas nacen con campos energéticos diferentes que se mantienen en 
constante cambio, generando en la salud de la persona cambios positivos o negativos según el comportamiento de los mismos (15).

Dentro de los metaparadigmas que son definidos como conceptos globales del fenómeno de interés por parte de la ciencia de enfermería son: la persona, la salud, el entorno y la enfermería. No obstante, han sido transformados a lo largo de la historia por las diferentes enfermeras dando lugar a la generación de modelos conceptuales (16). Para Rogers estos metaparadigmas que definen a la persona como un sistema abierto que interacciona constantemente con el entorno, se integran como campos de energía infinitos, irreductibles y pandimensionales específicos de cada ser humano (16).

Por otra parte, se puede decir que el objetivo de la teoría de Martha E. Rogers es promover una interacción de armonía entre el individuo y su entorno; es decir, que las enfermeras y enfermeros pueden guiarse y prestar cuidados individualizados, dirigidos a los patrones unidireccionales que componen el entorno de la persona y generen intervenciones que ayuden a la recuperación durante el proceso enfermedad (17).

Según la crítica científica la teoría de Martha Rogers ofrece conocimiento específico a la profesión de enfermería; además, se la ve como una teoría de evolución acelerada de correlatos rítmicos de cambio y de fenómenos paranormales (18). Los científicos hablan de la claridad de la teoría, aunque críticas tempranas hacían alusión a lo difícil que resultaba entender los principios de dicha teoría y la inadecuación de las herramientas de medida, pero que en la actualidad estas ideas demuestran claridad al ser aplicadas por la enfermería con seres humanos de cualquier edad (18).

Los estudios y trabajos realizados por la teoría de Rogers han sido de mucha importancia para aclarar conceptos y relacionar aspectos, pero aun así varios autores los categorizan como complejos. Actualmente se considera le da al hombre y su naturaleza principios y supuestos, haciéndolo sencillo a la hora de ponerlo a prueba por parte de enfermeras investigadoras (19). La teoría de Rogers es abstracta, generalizable y poderosa, con un objetivo que ofrece el marco de desarrollo del saber de la enfermería (19). Como ya se mencionaba anteriormente, esta teoría abarca el concepto de persona como ser único y permite valorar la relación individuo-entorno. Esta característica genera herramientas de medición que son importantes aportes para la profesión y que facilitan su desarrollo práctico.
Conviene subrayar que la importancia que tiene esta teoría es indispensable, pues comprende la evolución humana respecto al perfeccionamiento del hombre. Esta ciencia presenta un universo de sistemas abiertos que sirven para identificar nuevos paradigmas respecto a la enfermería como ciencia. Esto es que la teoría de Rogers presenta una relación lógica entre el saber moderno científico que alimenta la aparición de teorías nuevas que explican la comprensión del ser humano como sujeto unitario (19).

La enfermería moderna cuenta con instrumentos variados para la valoración de cada uno de los tiempos, momentos y condiciones en los que se encuentre la persona. Dentro de estos instrumentos de investigación se cuenta con un formato de valoración del campo de energía humano, que ayuda a registrar los hallazgos con la valoración del campo de energía humano direccionando el tacto terapéutico en donde se localice la alteración del campo, en un diagrama corporal con la fuerza e intensidad del campo en escalas analógicas (19). Durante la revisión bibliográfica se buscaron instrumentos acordes a esta investigación, que sirvieran para evaluar bajo la teoría de Rogers el comportamiento de la administración de medicamentos como tratamiento terapéutico que pueden generar cambios en la relación entorno-paciente. En la literatura se encontraron métodos en los que se evalúa la adherencia de los medicamentos categorizada en modelos, tipo de encuestas que realizan los profesionales de la salud, quienes tienen como tarea en su práctica profesional la administración o prescripción de medicamentos (Medicina y Enfermería) (20).

En la actualidad se cuenta con varios instrumentos de valoración que las enfermeras han desarrollado mediante la investigación e implementación de teorías sobre enfermería, que ayudan a valorar al individuo de forma global. Uno es el proceso de enfermería, herramienta que ayuda al enfermero a crear procesos de cuidados íntegros que generan intervenciones guiadas hacia la necesidad del paciente. Esta herramienta provee ciertas fases de desarrollo en las que se da una valoración inicial con la recolección de datos para la creación de metas y actividades; posteriormente se procura la solución de dicha necesidad que afecte directa o indirectamente a la persona (21). Otro de los grandes instrumentos de valoración con los que se cuenta en la enfermería moderna y que ha sido de suma importancia para el desarrollo práctico, son los patrones funcionales de Marjory Gordon. Estos patrones son una forma de guía agrupados que crean una entidad que se transforma con el pasar 
del tiempo y que representan al ser humano de forma integral; los mismos están categorizados por sistemas que van desde el más bajo hasta alta complejidad (22). Dentro de los instrumentos para evaluar la adherencia terapéutica según la farmacocinética y farmacodinamia en el artículo "Métodos para medir la adherencia terapéutica", se tienen métodos directos e indirectos que miden la adherencia del medicamento determinando el potencial de acción del fármaco ligado a la respuesta fisiológica del cuerpo humano (23).

Se debe precisar como dice Rogers que hay que continuar estudiando y profundizando, para que los profesionales de la enfermería logren brindar cuidado, teniendo en cuenta la totalidad de las dimensiones de la persona. Rogers con sus principios, conceptos y postulados vislumbra un camino para continuar reforzando el patrón de conocimiento desde la perspectiva investigativa (23). De ahí que la enfermería deba estar basada en la evidencia, para relacionar la teoría con la práctica; además, hay que promover el diálogo sobre el conocimiento, que para este caso consiste en determinar la respuesta humana frente a unos medicamentos especiales(23). En consonancia con lo planteado anteriormente, las enfermeras y enfermeros deben reflexionar sobre que se está construyendo desde la profesión para conocer cómo evoluciona el ser humano a partir de los principios de homeodinámicos y el concepto de la pandimensionalidad de la teoría de los seres humanos unitarios (24). Al respecto, Koffi's realizó un estudio basado en teoristas que le permitieran revolucionar el conocimiento; sin embargo, resalta que se debe ser crítico y reflexivo a partir de estas premisas universales, para poder competir o debatir en el ámbito del saber y asimismo tener una distinción de otros profesionales (25).

Para Rogers, la importancia de esta teoría está en ver al sujeto de cuidado como una persona única; es decir, tener una visión unitaria y optimista que sirva como guía para la enfermería, la investigación y la innovación en un mundo globalizado que está en constante helicidad o evolución (26). En Colombia, la organización colegial tiene como referente a Rogers, considerando que ella cambió el uso del concepto de hombre unitario por ser humano unitario para eliminar toda referencia al género; es por tanto, un ser que requiere ser tratado como único y enfocar los cuidados de manera individualizada no generalizada (27). La metodología de Rogers es dialéctica y no operacional, organiza el universo por partes, corresponde a una teoría deductiva y lógica. Esta autora utiliza la relatividad, la teoría general de sistemas y la teoría electrodinámica de la vida (28).

Con respecto a esta investigación este instrumento es necesario, por tanto en la siguiente fase se hace su validación para determinar los cambios necesarios si los hay y así fortalecer el cuidado en la administración de medicamentos desde el momento en que entran al organismo hasta la evaluación de sus efectos adversos. Además deben evaluarse los indicadores de la calidad en la atención (29). Con base en lo anterior en el Ministerio de Salud y la Protección social se ha construido una guía para identificar cuáles son las fallas más comunes y cuáles los factores que contribuyen o favorecen la aparición de errores en la administración de medicamentos (30).

De acuerdo con lo anterior, este instrumento de valoración siguiendo los lineamientos de la teoría de Martha E. Rogers no sólo permitirá medir la respuesta fisiológica terapéutica del medicamento, sino que también facilitará la identificación de los cambios que se puedan observar en el individuo durante su proceso de enfermedad, que afecten o mejoren el equilibrio armónico de los campos energéticos según la teoría de Rogers, medidos por principios homeodinámicos y el concepto de pandimensionalidad. Esto es, que se ha demostrado la innovación y la necesidad de su implementación en la enfermería moderna. Con este instrumento se podrá brindar cuidados de enfermería más íntegros que respondan y den solución eficaz a las necesidades de salud de la persona. En consonancia con esta construcción es necesaria en una fase posterior, la validez del instrumento en la práctica profesional (31).

\section{Conclusiones}

- Para Martha E. Rogers el individuo es un todo único que necesita procesos de enfermería individuales que satisfagan las necesidades implicadas en el desequilibrio de un campo energético perteneciente a los principios hemodinámicos que ella establece. En concordancia con el resultado de la valoración de la persona en todos sus campos o dimensiones se podrán brindar intervenciones para armonizar los principios homeodinámicos y el concepto de pandimensionalidad necesarios para recuperar la energía del ser humano y mejorar la relación con el entorno; todo esto a partir de la premisa de diálogo entre el sujeto, la familia y el cuidador. 
- Precisamente la administración de medicamentos como actividad única y propia del profesional de la enfermería requiere de una valoración óptima que ayude a determinar la percepción del sujeto de cuidado, ante ciertos medicamentos especializados que generan rápidamente efectos positivos o negativos, para redireccionar el cuidado si fuese necesario; de ahí la importancia de evaluar los principios homeodinámicos y las diferentes dimensiones del ser humano, en su concepto de pandimensionalidad.

- Esta revisión y construcción del instrumento permitirá a los estudiantes valorar al sujeto de cuidado desde una perspectiva teórica para comprender sus principios. De igual forma se podrá aplicarlo en intervenciones diarias de los profesionales de enfermería; siempre en función de la planificación de los cuidados integrales, valorando la helicidad (evolución en su estado) y la resonancia (la respuesta del sujeto de cuidado ante los efectos de los medicamentos). Asimismo, se tendrá en cuenta la pandimensionalidad (la armonía con su entorno o realidad desde las diferentes dimensiones, física, emocional y las relaciones con la familia y cuidadores)

- En la actualidad se cuenta con muchos instru- mentos de valoración que son herramientas fundamentales para el profesional de enfermería durante el desarrollo práctico de su profesión. Desde este punto de vista, la creación en esta investigación de nuevos instrumentos como el que se presenta, reforzarán las bases científicas sobre la enfermería, fortaleciendo y guiando el cuidado por un sendero integrado y humanizado que permita concretar una teoría tan abstracta a la práctica.

- Cabe señalar que la claridad y sencillez del instrumento que se propone en este estudio permite que el enfermero en formación lo aplique de forma veraz, poniendo en práctica su pensamiento crítico y reflexivo para la propuesta de intervenciones que ayuden a la persona a alcanzar su potencial máximo en salud. Como se ha demostrado se basa en la teoría de Rogers, generando procesos de enfermería que sean no solo humanizados sino de calidad.

\section{Conflicto de intereses}

Los autores no declaran conflicto de interés

\section{Referencias Bibliográficas}

1. Ley 266 de 1996. Colombia. Congreso de Colombia. 1996.

2. Cisneros F. Introducción a los modelos y teorías de Enfermería [Pregrado]. Universidad del Cauca; 2002.

3. Wright B. Roger's Science of Unitary Human Beings. Nursing Science Quarterly. 2006;19(3):229-230. https:// doi.org/10.1177\%2F0894318406289888

4. Sotelo-Sánchez J. Instrumentos de valoración del estado de salud en traumatología y cirugía ortopédica. Science Direct. 2004; 48(4):304-314. https://doi.org/10.1016/S1888-4415(04)76224-4

5. República de Colombia. Ministerio de Salud. Resolución Número 8430 de 1993, octubre 4, por la cual se establecen las normas científicas, técnicas y administrativas para la investigación en Salud. [Internet]. 1993 [consultado 20 de abril de 2019]; Disponible en: https://www.minsalud.gov.co/sites/rid/Lists/BibliotecaDigital/RIDE/DE/DIJ/RESOLUCION-8430-DE-1993.PDF

6. Sampieri HR. Metodología de la investigación. 6th. ed. México: McGRAW W-HILL; 2014.

7. Jin-Naa S, Ryang-Chunga C, Hyun-Chob Y, Kyeongman-Jeona C, Young-Suha G, et al. La escala de vasoactivos inotrópicos como predictora de mortalidad de adultos con shock cardiogénico tratados con y sin ECMO. Revista Española de Cardiología 2019; 72(1):40-47.

8. García-Canales A, Pena-Juárez RA, Sandoval-Franco LM. Vasopresores e inotrópicos: uso en pediatría. Archivos Cardiología México 2018; 88(1):39-50.

9. Gutiérrez-García A, Sánchez-Ocampo EM. Uso de las principales drogas inotrópicas, vasoactivas y vasodilatadoras en el perioperatorio. 2016; 39(1):218-222. 
10. Lipszyc F. Inotrópicos y vasopresores en el shock cardiogénico. Sociedad Argentina de Cardiología 2014; 10(1):78-88.

11. Perafán M. Inotrópicos y vasopresores en cirugía cardiovascular «un mal necesario». Revista Colombiana de Cardiología 2014; 21(4):264-265.

12. Adams MP, Holland LN, Bostwick PM. Farmacología para enfermería. Un enfoque fisiopatológico. Editorial Gea, Consultoría editorial, S.L; 2009. p 409-423.

13. Garnica-Camacho CE, Rivero-Sigarroa E, Domínguez-Cherit G. Choque cardiogénico: de la definición al abordaje. Medicina Critica 2019; 33(5):251-258.

14. Achury DM, Achury LF, Rodríguez SM, Díaz Álvarez JC. Fundamentos enfermeros en el proceso de administración de medicamentos. Editorial Pontificia Universidad Javeriana; 2013. P 70-80.

15. Rogers M. An Introduction to The Theoretical Basis of Nursing. 4th. ed. New York: New York University; 1976.

16. Huamán-Salazar N, Miranda-Alvarado A, Vásquez-Ramírez J, González-Mesa B, García-Padilla AA, Arteaga león-Livia. Utilización de Modelos y Teorías de Enfermería de Escuelas del Ser Humano Unitario y Caring en Servicios del Hospital María Auxiliadora. Revista Científica de Ciencias de la Salud [internet]. 2018 [consultado el 23 ov 2019]; 8(1):21-18. https://doi.org/10.17162/rccs.v8i1.978

17. Raile Alligood M, Marriner Tomey A. Modelos y teoría en enfermería. 9th. ed. Barcelona: Elsevier; 2014.

18. Smrit A. Integration of nursing theories in practice. International Journal of Nursing Science Practice and Research. 2015; 1(1): 8-12.

19. Gonzales MD, Palacio MJ, Oliver CA, de la Fuente FN, Mariscal BC, Corral RAM, et al. Enfermería conceptos generales. 1st. ed. Bogotá: Ediciones de la U; 2015.

20. Neus PP, Isabel VM. Métodos para medir la adherencia terapéutica. Revista Ars Pharm. 2018; 59(3): 163-172.

21. Phillips Jhon R. Rogerian Nursing Science and Research: A Healing Process for Nursing 2000; 13(3):196-201.

22. Ruíz-Gómez CE, Alfonso LM, Bernal-Espejo LM, Salgado-Suarez M. El proceso de enfermería. Método integrado del cuidado con calidad humana. 1st. ed. Bogotá: Salud Areandina; 2014.

23. Reed GP. The Force of Nursing Theory-Guided Practice. Nurs Sci Q. 2006; 19(3): 225.

24. Wright WB. The Evolution of Rogers' Science of Unitary Human Beings: 21ST Century Reflections 2007; 20(1):64-67.

25. Koffi K. The Universal Conceptualization of Nursing from the Two Nursing Disciplinary Scientific Revolutions 2017; 23(2):13-23.

26. Baumann-Steven L, Wright-Stephan G. A Science of Unitary Human Beings Perspective of Global Health Nursing 2014; 27(4): 324-328.

27. Organización Colegio de Enfermería. Martha Rogers: Su teoría para Enfermería. [Internet]. [Consultado 20 oct 2019]. Disponible en: https://www.oceinfo.org.co/index.php/difusion/noticias/60-martha-rogers-su-teoria-para-enfermeria

28. Duque-Henao MA. Seres Humanos Unitarios Martha Rogers. [Internet]. [Consultado en mayo 2020]. Disponible en: http://modelosenfermerosucaldas.blogspot.com/2013/06/seres-humanos-unitarios.html

29. Henao-Nieto DE, Giraldo-Villa A, Yepes-Delgado CE, Instrumentos para evaluar la calidad percibida por los usuarios en los servicios de salud. Revista Gerencia y políticas de Calidad.; 2017.

30. Ministerio de Salud. Mejorar la seguridad en la utilización de medicamentos paquetes instruccionales. Guía técnica "buenas prácticas para la seguridad del paciente en la atención en salud". 2008 [Internet]. [Consultado en enero 2020]. Disponible en: https://www.minsalud.gov.co/sites/rid/Lists/BibliotecaDigital/RIDE/DE/CA/ seguridad-en-la-utilizacion-de-medicamentos.pdf

31. Cervantes H. V. Interpretación del coeficiente Alpha de Cronbach. Avances en Medición. 2005; 3(28):1-20. 